\title{
EXAMPLES OF CENTRALIZERS OF AUTOMORPHISM GROUPS
}

\author{
ALEXANDRE TURULL
}

\begin{abstract}
If $A G$ is a finite solvable group with $(|A|,|G|)=1$ and $G \triangleleft A G$, we explore the relationship between the Fitting height of $G$, the composition length of $A$ and the structure of $C_{G}(A)$. For any $A$, we give examples where the difference between the Fitting height of $G$ and that of $C_{G}(A)$ is exactly twice the composition length of $A$.
\end{abstract}

All groups in this paper are assumed to be finite. Let $A$ be a solvable group and assume that $A$ acts as automorphisms of a solvable group $G$ such that $(|A|,|G|)=1$. Then it is well known that the structure of $C_{G}(A)$ is intimately related to that of $G$. For example, it is known [3] that in that case, if $h$ denotes Fitting height and $k$ composition length, then $h(G) \leq 2 k(A)+h\left(C_{G}(A)\right)$. The purpose of this paper is to analyze to what extent this and related results are the best possible. We prove

THEOREM A. Let $A$ be any solvable group and $h$ any integer greater than zero. Assume further that $p$ and $q$ are distinct primes not dividing $|A|$. Then there exists a (solvable) $\{p, q\}$-group $G$ and an action of $A$ on $G$ as automorphisms such that $h\left(C_{G}(A)\right)=h$ and $h(G)=2 k(A)+h$.

The special case of Theorem A where $k(A)=1$ is due to E. C. Dade and appears in $[\mathbf{2}]$. We also prove the following, where $F$ denotes Fitting subgroup.

THEOREM B. Given any two solvable groups $A$ and $C$ such that $(|A|,|C|)=1$, and any prime $p \nmid|A||F(C)|$, there exists a solvable group $G$ and an action of $A$ on $G$ by automorphisms such that $(|A|,|G|)=1, F\left(C_{G}(A)\right)=O_{p}\left(C_{G}(A)\right)$, $C_{G}(A) / O_{p}\left(C_{G}(A)\right) \simeq C$ and $h(G)=2 k(A)+h\left(C_{G}(A)\right)$.

If $A \neq 1$ and $C_{G}(A)$ has all its Fitting factors Abelian, it follows from $[\mathbf{3}$, Theorem 3.1] that $h(G) \leq 2 k(A)+h\left(C_{G}(A)\right)-1$. One cannot therefore prescribe $C_{G}(A)$ arbitrarily if we want to have $h(G)=2 k(A)+h\left(C_{G}(A)\right)$. Furthermore, we have by [3, Remark 3.3], that $h(G) \leq k(A)+h\left(C_{G}(A)\right)$ if all Fitting factors of $G$ are pairwise relatively prime. The special case of the next theorem where $C=1$ is due to Gross [1].

TheOREM C. Let $A$ and $C$ be solvable groups and assume $(|A|,|C|)=1$. Then there exists a solvable group $G$ and an action of $A$ on $G$ by automorphisms such that $(|A|,|G|)=1, C_{G}(A) \simeq C$ and $h(G)=k(A)+h\left(C_{G}(A)\right)$. Furthermore, if all ascending Fitting factors of $C$ are pairwise of relatively prime order, $G$ can be chosen with the same property.

Given a solvable group $C$ and a positive integer $a$ define

$$
m(a, C)=(1 / a) \max \left\{h(G)-h\left(C_{G}(A)\right)\right\}
$$

Received by the editors April 7, 1983.

1980 Mathematics Subject Classification. Primary 20D45; Secondary 20F28.

(C) 1984 American Mathematical Society $0002-9939 / 84 \$ 1.00+\$ .25$ per page 
where $A$ and $G$ range over all solvable groups such that $k(A)=a,(|A|,|G|)=1, A$ acts on $G$ by automorphisms and $C_{G}(A) \simeq C$. We know that $1 \leq m(a, C) \leq 2$, the first inequality by Theorem $\mathrm{C}$ and the second by [3, Corollary 3.2]. It is a well-known conjecture that $\sup _{a \geq 1}(m(a, 1))=1$. It follows from Theorem $\mathrm{A}$ that for infinitely many choices of $C, \sup _{a \geq 1}(m(a, C))=2$. Nevertheless, the following seems plausible.

CONJECTURE. For any solvable $C, \lim _{a \rightarrow \infty}(m(a, C))=1$.

2. Proofs. In this section we construct the relevant examples.

LeMma 2.1. Let $B$ be a subgroup of a group $A$. Assume that $B$ acts on a group $G$. Then there exists a group $G^{*}$ isomorphic to the direct product of $|A: B|$ copies of $G$ and an action of $A$ on $G^{*}$ such that $C_{G^{*}}(A) \simeq C_{G}(B)$, and if $B \triangleleft A$ then $C_{G^{*}}(B)$ is the direct product of $|A: B|$ copies of $C_{G}(B)$.

ProOF. This, of course, is just induction. For example, we may imbed $A$ in the usual way by a Frobenius map (see for example [4, Proposition 1.6]) into the wreath product $S_{n} \sim B$, where $n=|A: B|$ and $S_{n}$ is the symmetric group. This provides a natural action of $A$ on $G^{*}$. If $x \in C_{G^{*}}(A)$ then it is centralized by $B \times 1 \times \cdots \times 1$. By taking conjugates we get that $x$ is centralized by the normal subgroup $B^{|A: B|}$ of $S_{n} \sim B$. But then $A$ acts on $x$ just by transitively permuting the indices, so all the coordinates of $x$ are equal and are centralized by $B$. Hence $C_{G^{*}}(A) \simeq C_{G}(B)$ as desired. If $B \triangleleft A$ then $B$ is mapped into $B^{|A: B|}$ by the Frobenius map and $C_{G^{*}}(B)=\left[C_{G}(B)\right]^{|A: B|}$. This proves the lemma.

LEMMA 2.2. Assume that $A$ is a group and $B \triangleleft A$. Assume further that $A$ acts on two groups $G_{1}$ and $G_{2}$ of order $g_{1}$ and $g_{2}$, respectively, and that $B$ centralizes $G_{1}$. Let $G_{1} \sim G_{2}$ denote the wreath product of order $g_{1} g_{2}^{g_{1}}$. Then $A$ acts in a natural way on $G_{1} \sim G_{2}$, the action of $A$ on the natural image of $G_{1}$ in $G_{1} \sim G_{2}$ is equivalent to that of $A$ on $G_{1}$ and $C_{G_{2}} g_{1}(B)=\left[C_{G_{2}}(B)\right]^{g_{1}}$.

Proof. $G_{1} \sim G_{2}$ is the set of ordered pairs $(\pi, f)$ where $\pi$ is a permutation of $G_{1}$ obtained by left multiplication by some fixed element of $G_{1}$ and $f$ is a function from $G_{1}$ to $G_{2}$.

$$
(\pi, f)\left(\pi^{\prime}, f^{\prime}\right)=\left(\pi \circ \pi^{\prime},\left(f \circ \pi^{\prime}\right) \cdot f^{\prime}\right)
$$

where $\circ$ denotes composition of functions, and - product by components. We think of the elements of $A$ as permutations of $G_{1}$ and $G_{2}$, and define, for $a \in A$,

$$
a((\pi, f))=\left(a \circ \pi \circ a^{-1}, a \circ f \circ a^{-1}\right) .
$$

For any $g \in G_{1}$ let $\pi$ be its corresponding Cayley permutation and set $\sigma(g)=$ $(\pi, 1)$, where $1(x)=1$ for all $x \in G_{1}$. Then

$$
a(\sigma(g))=\left(a \circ \pi \circ a^{-1}, a \circ 1 \circ a^{-1}\right)=\left(a \circ \pi \circ a^{-1}, 1\right)
$$

and if $x \in G_{1},\left(a \circ \pi \circ a^{-1}\right)(x)=a(g) x$. So the action of $A$ on the natural image of $G_{1}$ in $G_{1} \sim G_{2}$ is equivalent to the action of $A$ on $G_{1}$.

Since $a$ provides an automorphism of $G_{2}$ it is easy to verify that $a$ provides an automorphism of $G_{1} \sim G_{2}$ and that this gives a representation of $A$ as automorphisms of $G_{1} \sim G_{2}$. 
If $b \in B$ then $b$ acts as the trivial permutation on $G_{1}$ and it acts on (Id, $f$ ) by $b((\operatorname{Id}, f))=(\operatorname{Id}, b \circ f)$. Whence we get $C_{G_{2}} g_{1}(B)=\left[C_{G_{1}}(B)\right]^{g_{1}}$. This concludes the proof of 2.2 .

We define as usual $F_{0}(G)=1$ and $F_{i}(G) / F_{i-1}(G)=F\left(G / F_{i-1}(G)\right)$ for all positive integers $i$.

LEMMA 2.3. Let $A$ be any solvable group. Let $h=k(A)$ and let $p_{i}, i=1, \ldots, h$, be a finite sequence of primes. Let $p$ be a prime such that $p \nmid p_{1} \cdots p_{h}|A|$, and assume $\left(p_{1} \cdots p_{h^{\prime}}|A|\right)=1$. Then there exists a solvable group $G$ and an action of $A$ on $G$ by automorphisms such that:

1. $\pi(G)=\left\{p, p_{i}: i=1, \ldots, h\right\}$.

2. $h(G)=2 h$.

3. $F_{i}(G) / F_{i-1}(G)$ is an elementary Abelian $p_{j}$-group if $i=2 j$ and an elementary Abelian $p$-group if $i$ is odd.

4. If $p_{i} \neq p_{i+1}, i=1, \ldots, h-1$, and $H$ is a Hall $p^{\prime}$-subgroup of $G$ then $h(H)=h$ and $F_{i}(H) / F_{i-1}(H)$ is an elementary Abelian $p_{i}$-group, $i=1, \ldots, h$.

5. $C_{G}(A)$ is a p-group.

Proof. Assume false. Let $A$ be a counterexample with $k(A)$ minimal. Assume first that $k(A)>1$. Let then $B$ be a normal subgroup of $A$ such that $k(B)+1=$ $k(A)$. By induction we may find $G_{1}$ satisfying the conditions for $p_{1}, \ldots, p_{h-1}$ and $B$, and $G_{2}$ satisfying them for $p_{h}$ and $A / B$. By Lemma 2.1 we may assume $A$ acts on $G_{1}$ and, by Lemma 2.2, $A$ acts on the wreath product $W=G_{2} \sim G_{1}$, and it is easy to verify that $W$ satisfies properties $1-5$. So since the result is clear if $k(A)=0$, we have $k(A)=1$. Let $A$ act by multiplication on the additive group $F$ of the extension of $\mathbf{F}_{p_{1}}$ by a primitive $|A|$ th root of 1 . Let $M$ be a faithful $\mathbf{F}_{p} A F$ module. Then set $G=F M$. It is clear that $G$ satisfies 1-5. This contradiction concludes the proof of Lemma 2.3.

ProOF OF THEOREM A. Let $D$ be a solvable $\{p, q\}$-group such that $h(D)=h$ and $F(D)=O_{p}(D)$. Let $G$ satisfy the condition of Lemma 2.3 with the sequence $p_{i}=q, i=1, \ldots, k(A)$. Then $W=D \sim G$ satisfies the conditions of Theorem A by Lemma 2.2 , since $C_{W}(A)$ is an extension of $D$ by a $p$-group and $h\left(C_{W}(A)\right)=h$.

Proof OF THEOREM B. Let $D=C \sim \mathbf{Z}_{p}$ and choose a prime $q$ such that $q \nmid p|A|$. Use the construction in the proof of Theorem $A$.

ProOF OF THEOREM C. Take $p_{1}, \ldots, p_{h}, h=k(A)$ a sequence of primes such that $p_{i} \neq p_{i+1}, i=1, \ldots, h=1,\left(p_{1} \cdots p_{h},|A|\right)=1$ and $p_{h} \nmid|F(C)|$. Let $H$ be an $A$-invariant Hall $p^{\prime}$-subgroup of the group $G$ given by Lemma 2.3. Take $C \sim H$. By Lemma 2.2 we get the theorem.

\section{REFERENCES}

1. Fletcher Gross, A note on fuxed-point-free solvable operator groups, Proc. Amer. Math. Soc. 19 (1968), 1363-1365.

2. Hans Kurzweil, $p$-Automorphismen von auflösbaren $p^{\prime}$-gruppen, Math Z. 120 (1971), 326-354.

3. Alexandre Turull, Fitting height of groups and of fixed points, J. Algebra 86 (1984), 555-556.

4. __ Supersolvable automorphism groups of solvable groups, Math. Z. 183 (1983), 47-73.

Department of Mathematics, University of Miami, Coral Gables, Florida 